Surf ace Pl asmon Ant enna wi th Gol d Li ne and Space Grat ing for Enhanced Vi si bl e Li ght Det ect i on by a Si I i con- on- I nsul at or Net al Oxi de Semi conduct or Phot odi ode

\begin{tabular}{|c|c|}
\hline 著者 & Sat oh H r oaki, I nokawa H r oshi \\
\hline $\begin{array}{l}\text { jour nal or } \\
\text { publ i cat } i \text { on title }\end{array}$ & I EEE Tr ansact i ons on Nanot echnol ogy \\
\hline vol une & 11 \\
\hline nunber & 2 \\
\hline page $r$ ange & $346-351$ \\
\hline year & 2011- 11- 15 \\
\hline 出版者 & I EEE Nanot echnol ogy Counci I \\
\hline 権利 & 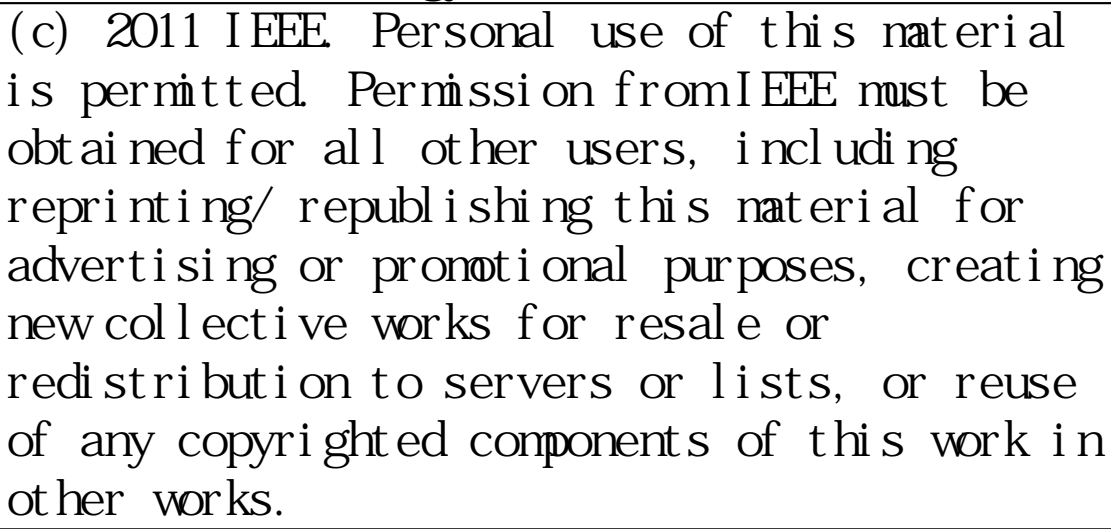 \\
\hline URL & ht t p: //hdl . handl e. net /10297/7958 \\
\hline
\end{tabular}




\title{
Surface Plasmon Antenna with Gold Line and Space Grating for Enhanced Visible Light Detection by Silicon-On-Insulator Metal-Oxide-Semiconductor Photodiode
}

\author{
Hiroaki Satoh, Member, IEEE, and Hiroshi Inokawa, Member, IEEE
}

\begin{abstract}
A gold (Au) line and space (L/S) grating antenna for the silicon-on-insulator metal-oxide-semiconductor (SOI-MOS) photodiode is investigated. The SOI-MOS photodiode has the advantages such as high-speed operation, large voltage gain per electric charge, and small dark current. However, its light sensitivity is usually very low due to the small volume of light absorption. In order to enhance the light sensitivity, a surface plasmon (SP) antenna is adopted. The SP antenna is constructed with periodic metallic structure, and can convert the incident light to optical near-field around the surfaces of the antenna. By replacing the MOS gate with the SP antenna, it becomes possible to enhance the light absorption while keeping the advantages of SOI-MOS photodiode. This paper focuses on the visible light absorption in the SOI-MOS photodiode with the SP antenna. The structural conditions for high absorption efficiency and single-peaked spectroscopic characteristics, polarized light response, and resonance mechanisms are clarified by electromagnetic simulations using the finite-difference time-domain (FDTD) method.

Index Terms - surface plasmon antenna, enhanced light absorption, SOI-MOS photodiode, spectroscopic response, polarized light response.
\end{abstract}

\section{INTRODUCTION}

$\mathrm{P}$ hotodetector performance trade-off between the operation speed and the light sensitivity is imposed by the volume of light absorber. For silicon photodetector, silicon-on-insulator (SOI) is expected to have advantages of high-speed operation and large voltage gain per electric charge because of the small parasitic capacitances. However, the light sensitivity is usually not high because SOI layer compatible with integrated circuits is not thick enough to attain efficient light absorption. For better performance, an additional mechanism is needed to enhance the light sensitivity while keeping the above SOI advantages.

After the Ebbesen's discovery of extraordinary transmission through sub-wavelength hole array [1]-[3], the sub-wavelength

Manuscript received $* * * * * *, 2010$.

H. Satoh and H. Inokawa are with the Research Institute of Electronics, Shizuoka University, Hamamatsu 432-8011 Japan (corresponding author to provide phone: +81-53-478-1325; fax: +81-53-478-1651; e-mail: h-satoh@rie.shizuoka.ac.jp). optics have been applied to many optical fields because it can provide the transmission efficiency greater than predicted by the standard aperture theory, and overcome the fundamental diffraction limit. The phenomenon utilizes the optical near-fields arising from the SP waves that are strongly localized along the surfaces of periodic metallic structure. In the field of photodetector, SP phenomena have been used to enhance the light sensitivity via SP excitation in either metal nanoparticles [4] or gratings [5], [6] attached on semiconductor photodiodes. As one extension, silver (Ag) SP antenna with circular gratings (called bull's eye) has been proposed to enhance the light sensitivity of nanometer-scale high-speed Schottky photodiode operating at a near infrared wavelength [7]. In high-speed metal-semiconductor-metal (MSM) photodiodes, SP excitations in Au gratings have also been utilized [8], [9].

This paper discusses the enhanced light sensitivity (i.e. the light absorption) in SOI-MOS lateral p-i-n photodiode by $\mathrm{Au}$ $\mathrm{SP}$ antenna with L/S grating structure. The structural conditions for high absorption efficiency and single-peaked spectroscopic characteristics are clarified by electromagnetic simulations using the two-dimensional (2D) finite-difference time-domain (FDTD) method [10]. Resonance mechanisms for different absorption peaks are also discussed for understanding the photodiode. Finally, the response to polarized light is evaluated for different $\mathrm{L} / \mathrm{S}$ directions by three-dimensional (3D) simulation.

\section{DEVICE STRUCTURE AND SIMULATION CONDITIONS}

Figure 1 shows the schematic of the proposed MOS p-i-n photodiode with $\mathrm{Au} \mathrm{L} / \mathrm{S}$ grating on SOI substrate, and the 2D unit periodic structure for electromagnetic simulation. In this paper, it is assumed that the incident light is a plane wave and enters perpendicularly to the SOI-MOS photodiode with Au SP antenna. Generally, noble metals are used in SP excitations, because the real part of the permittivity in the visible and infrared wavelength range is negative. Ag is mostly used in the devices utilizing SP phenomena because of low optical losses. However, as $\mathrm{Au}$ is more durable to surface oxidation than $\mathrm{Ag}$, 


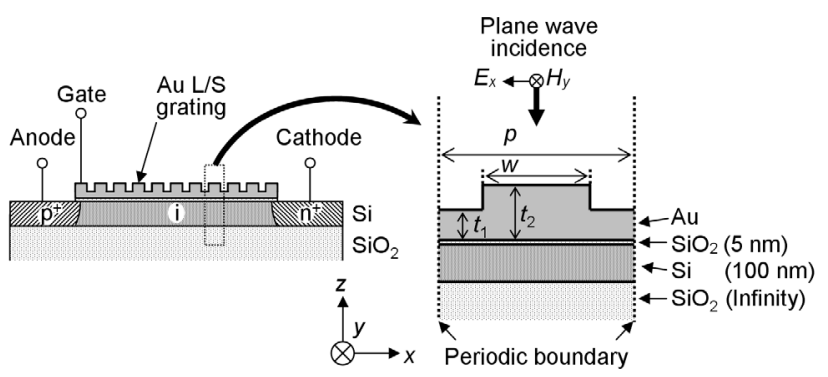

Fig. 1. A p-i-n photodiode with Au L/S grating SP antenna on SOI substrate and $2 \mathrm{D}$ unit structure for electromagnetic simulations. It is assumed that the structure is periodic infinitely in $x$-direction. Incident light is polarized as indicated by magnetic field $H_{y}$ and electric field $E_{x}$.

this paper deals with the grating made of Au. In order to simplify the analysis, the thickness of the lower silicon dioxide $\left(\mathrm{SiO}_{2}\right)$ layer [called buried oxide (BOX) layer] is assumed to be infinite. It has been well known that the SPs can be excited along the metal grating surface by the incident light almost without the reflection, as is manifested by the Wood's anomaly taking place in the grating structures. Consequently, intense non-radiative optical near-field can be generated along the grating surface. Furthermore, the optical near-field penetrates to the underneath silicon $(\mathrm{Si})$ layer, if the grating is very thin partially. By utilizing such optical near-field, the light absorption in the Si can be enhanced as will be described in the next section. Since the strength of the excited SP mode along the backside surface of Au SP antenna is exponentially decreased, the upper $\mathrm{SiO}_{2}$ thickness should be as thin as possible. On the other hand, this layer cannot be removed, because the electric isolation between the layers of $\mathrm{Au}$ and $\mathrm{Si}$ is required to use $\mathrm{Au} \mathrm{SP}$ antenna as a gate electrode. Since the direct tunneling current becomes conspicuous in $\mathrm{SiO}_{2}$ thinner than $5 \mathrm{~nm}$, the thickness of the upper $\mathrm{SiO}_{2}$ layer is fixed at $5 \mathrm{~nm}$. In actual fabrication, an adhesion material, such as titanium (Ti), should be inserted between $\mathrm{Au}$ and $\mathrm{SiO}_{2}$ for the structural stability. Although this paper focuses on the fundamental effects of SP excitation without the adhesion material, the presence of such a material would adversely affect the absorption efficiency, and, in the worst case, the efficiency would be reduced by one order of magnitude [12]. In the 2D FDTD electromagnetic calculations, the discretized interval and time step are $\Delta d(=\Delta x=\Delta z)=1 \mathrm{~nm}$ and $\Delta t=\Delta d /\left(2 c_{0}\right)=$ $1.67 \times 10^{-18} \mathrm{~s}$, respectively. Here, $c_{0}$ is the light speed in vacuum. The relative permittivities of 1.0 and 2.13 are used for vacuum and $\mathrm{SiO}_{2}$, respectively. In the cases of $\mathrm{Si}$ and $\mathrm{Au}$, the complex permittivities expressed by the Lorentz- and the Drude-type dispersions, respectively, are used for FDTD calculations. Specifically, the complex relative permittivity of $\mathrm{Si}$ is given by

$\varepsilon_{r}=\varepsilon_{\infty}+\frac{\left(\varepsilon_{s}-\varepsilon_{\infty}\right) \omega_{p}{ }^{2}}{\omega_{p}{ }^{2}+2 j \omega \delta_{p}-\omega^{2}}$,

where $\varepsilon_{\mathrm{s}}$ is the static or zero-frequency relative permittivity, $\varepsilon_{\infty}$ is the relative permittivity at infinite frequency, $\omega_{\mathrm{p}}$ is the frequency of pole pair, and $\delta_{\mathrm{p}}$ is the damping coefficient. In this

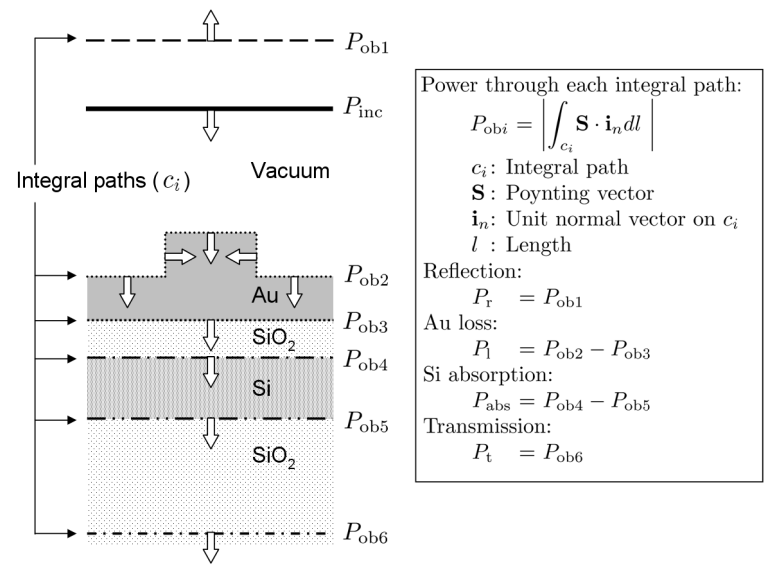

Fig. 2. Definition of integral paths in the analyzed structure and equations for obtaining power components.

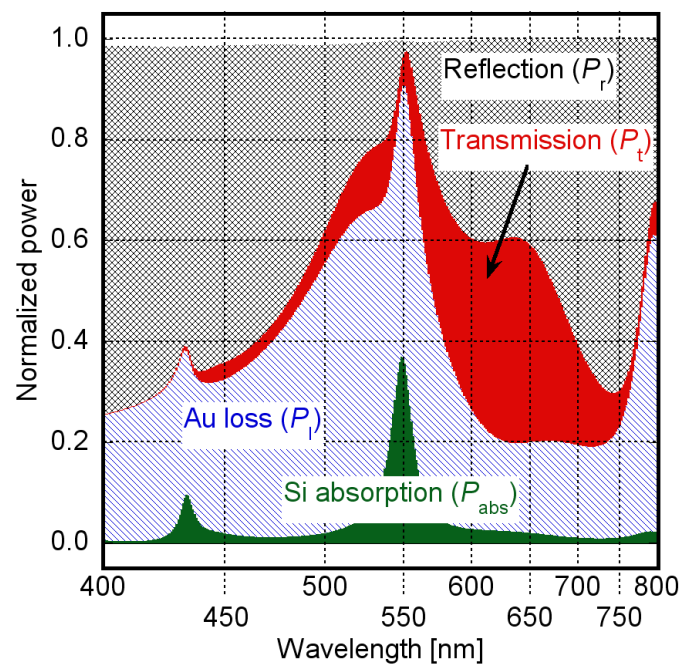

Fig. 3. Stacked area chart of power components as a function of incident wavelength. Grating period and width are $p=200 \mathrm{~nm}$ and $w=140 \mathrm{~nm}$, respectively. Au thicknesses are $t_{1}=30 \mathrm{~nm}$ and $t_{2}=80 \mathrm{~nm}$. Si thickness is $t_{\mathrm{SOI}}=$ $100 \mathrm{~nm}$.

paper, such parameters of $\mathrm{Si}$ are decided by fitting the experimental results provided by SOPRALAB [11] for visible light range. The Si parameters in FDTD calculations are $\varepsilon_{\mathrm{s}}=$ $12.7, \varepsilon_{\infty}=8.3, \omega_{\mathrm{p}}=5.145 \times 10^{15} \mathrm{rad} / \mathrm{s}$, and $\delta_{\mathrm{p}}=5.145 \times 10^{13}$ $\mathrm{rad} / \mathrm{s}$. On the other hand, the complex relative permittivity of $\mathrm{Au}$ is given by

$\varepsilon_{r}=\varepsilon_{\infty}+\frac{\omega_{p}{ }^{2}}{\omega^{2}-j \omega \gamma_{p}}$.

where $\omega_{p}$ is the Drude pole frequency, and $\gamma_{p}$ is the inverse of the pole relaxation time. We assume the Au parameters are $\varepsilon_{\infty}=$ $1, \omega_{\mathrm{p}}=1.027 \times 10^{16} \mathrm{rad} / \mathrm{s}$, and $\gamma_{\mathrm{p}}=2.83 \times 10^{14} \mathrm{rad} / \mathrm{s}$. These dispersion characteristics are expressed by piecewise-linear recursive-convolution (PLRC) method in FDTD formulation to attain high accuracy [10]. Furthermore, the boundary conditions for computational space in $x$-, and $z$-direction in Fig. 1 are periodic boundary, and absorbing with the perfectly matched layer (PML), respectively [10].

In order to obtain the sensitivity of photodetector, the power absorbed in Si relative to the incident one must be calculated. 


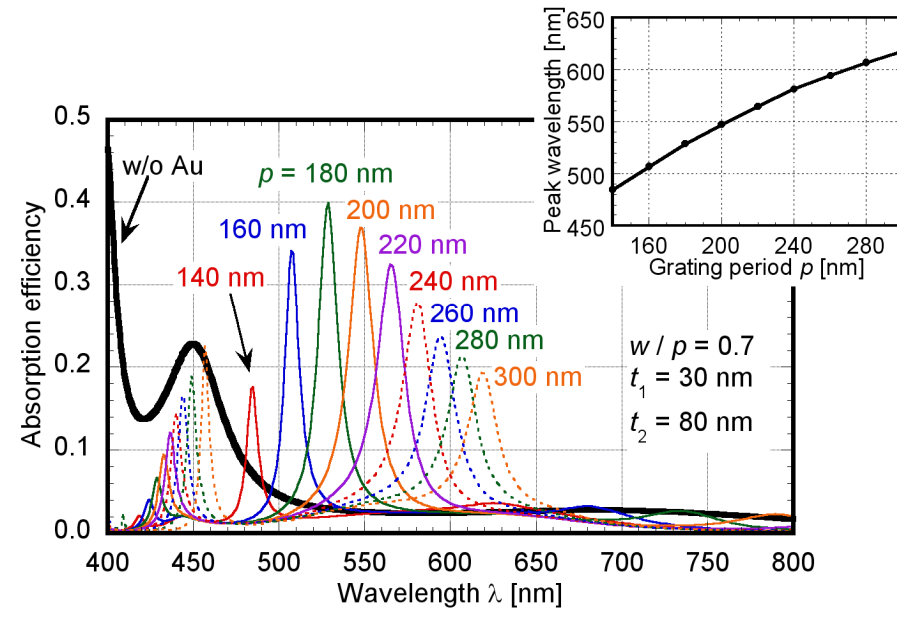

(a)

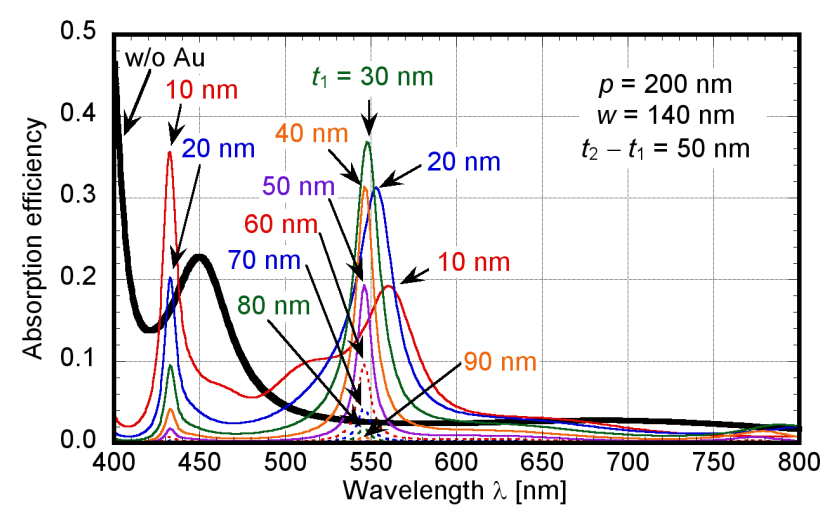

(c)

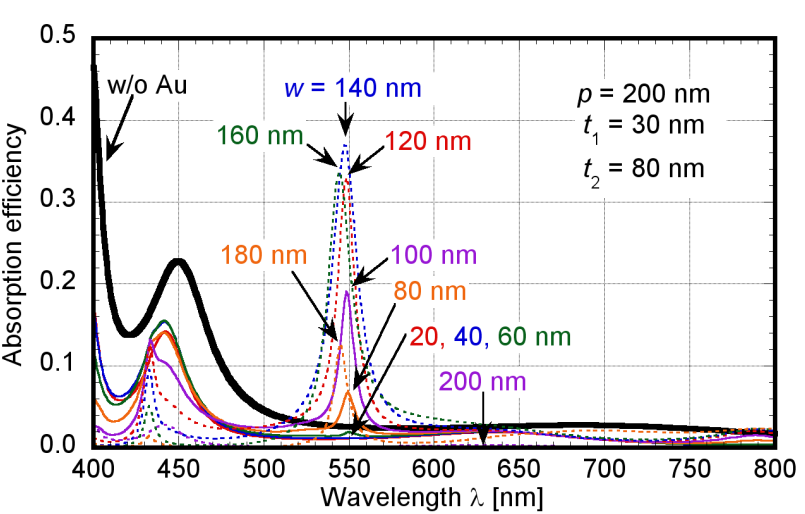

(b)

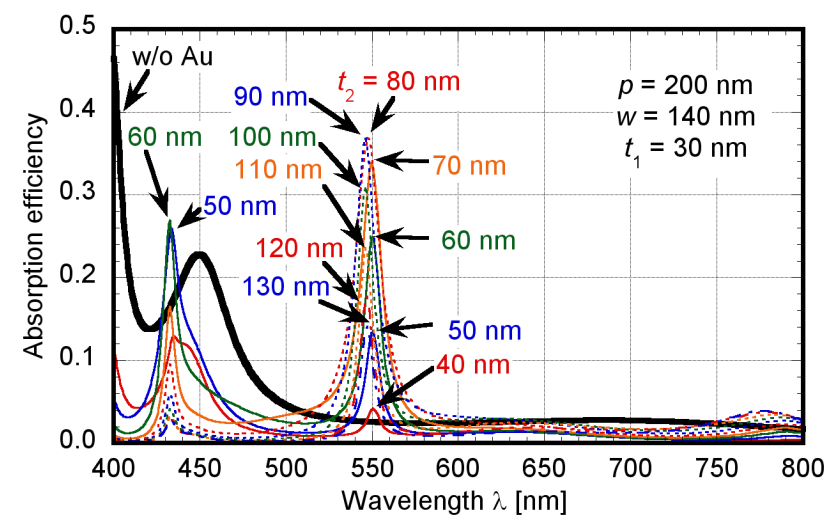

(d)

Fig. 4. Silicon absorption efficiencies $\left(P_{\text {abs }} / P_{\text {inc }}\right)$ with (a) grating period $p$, (b) grating width $w$, (c) thinner Au thickness $t_{1}$, and (d) thicker Au thickness $t_{2}$ as parameters. Silicon thickness is fixed at $t_{\mathrm{SOI}}=100 \mathrm{~nm}$.

Figure 2 shows the positions of integral paths and the equations for the power calculations. Power passing through each integral path is obtained by integrating Poynting vectors in the downward vertical direction. The $\mathrm{Si}$ absorption $P_{\text {abs }}$ can be calculated by subtracting the output power $P_{\mathrm{ob} 5}$ at the lower interface of Si from the input power $P_{\mathrm{ob} 4}$ at the upper interface as shown in this figure. The other ones such as reflection $P_{\mathrm{r}}$, transmission $P_{\mathrm{t}}$, and the power loss inside the Au $P_{1}$ are also calculated here. Note that the frequency (wavelength) response of these powers is obtained by performing the fast Fourier transformation (FFT) to the results of the Gaussian pulse response at $2^{19}$ sampling times in the FDTD calculation, because the FDTD solves the electromagnetic fields in the time domain. If these powers are shown in the stack as in Fig. 3, the fraction of each power converted from the incident light can be recognized. In this example, major SP resonance occurs, and the reflection and the transmission decrease at the wavelength $\lambda$ $=550 \mathrm{~nm}$. Therefore, the incident power is efficiently absorbed in $\mathrm{Si}$, but the $\mathrm{Au}$ conduction loss also becomes high simultaneously.

\section{RESULTS AND DISCUSSION}

\section{A. Enhanced Light Absorption by SP Antenna and Antenna Structure Optimization}

Figure 4 shows the dependence of absorption efficiencies on wavelength with various structural parameters. These figures include the absorption efficiency without $\mathrm{Au} \mathrm{L} / \mathrm{S}$ grating for comparison. The Si thickness is fixed at $t_{\mathrm{SOI}}=100 \mathrm{~nm}$. The peak wavelength can be varied by adjusting only the grating period $p$ as shown in Fig. 4(a). The ratio of grating period and grating width $w$ is fixed at $w / p=0.7$ in this case. We can see that the peak wavelength can be moved from 480 to $620 \mathrm{~nm}$ by simply increasing $p$. In other words, the Au L/S grating works as a filter whose peak wavelength can be tailored. Other structural parameters such as $w$, thinner Au thickness $t_{1}$, and thicker Au thickness $t_{2}$, barely change the peak wavelengths as shown in Fig. 4(b) to (d). Thus, these parameters are optimized for maximum absorption efficiency at around the wavelength of $550 \mathrm{~nm}$ with fixed $p=200 \mathrm{~nm}$. Since the optimized thinner $\mathrm{Au}$ thickness of our SP antenna is as thin as $30 \mathrm{~nm}$, there is a strong resonant coupling between SPs along the top Au surface and the waves in the underneath SOI layer. Therefore, this SP antenna is fundamentally different from the one in Ref. [8] 


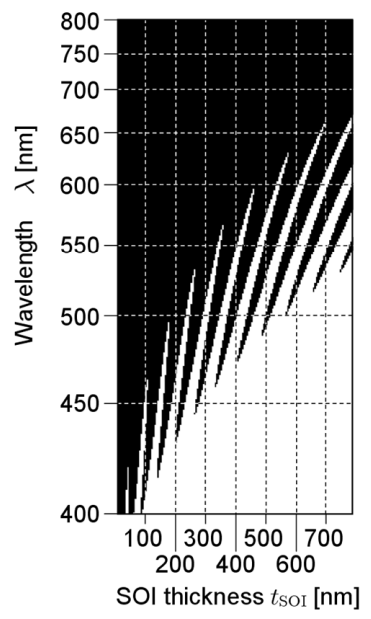

(a)

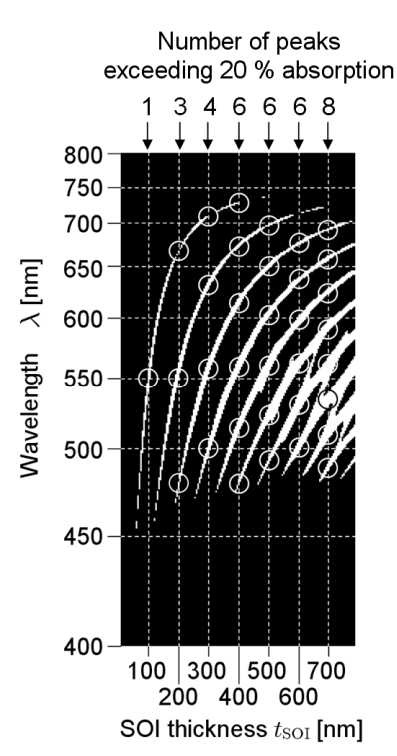

(b)

Fig. 5. Binary distribution of silicon absorption efficiency in the cases (a) without $\mathrm{Au}$ and (b) with $\mathrm{Au}$ in the plane of incident wavelength and SOI thickness. White area corresponds to the absorption exceeding $20 \%$. Grating period and width are $p=200 \mathrm{~nm}$ and $w=140 \mathrm{~nm}$, respectively. Au thicknesses are $t_{1}=30 \mathrm{~nm}$ and $t_{2}=80 \mathrm{~nm}$

which utilizes SPs propagating along the metal grating. As it is not straightforward to solve this problem theoretically, the FDTD calculations are very useful for the antenna optimization. For example, for the 550-nm-light detection, the absorption efficiency can be made maximum to $37 \%$ when $p=200 \mathrm{~nm}, w$ $=140 \mathrm{~nm}, t_{1}=30 \mathrm{~nm}$, and $t_{2}=80 \mathrm{~nm}$. This absorption efficiency is 14 times larger than the $2.6 \%$ in the case without the antenna. Here, it is interesting to note that the thin Au does not necessarily lead to higher absorption in Si as shown in Fig. 4(c) and (d). On the contrary, it is also shown in the same figures that the absorption efficiency at the shorter wavelength around $430 \mathrm{~nm}$ can be also enhanced when the $t_{1}$ and $t_{2}$ are very thin, specifically for the cases of $t_{1}=10 \mathrm{~nm}$ and $20 \mathrm{~nm}\left(t_{2}=60\right.$ $\mathrm{nm}$ and 70nm, respectively) in Fig. 4(c), and $t_{2}=50 \mathrm{~nm}$ and 60 $\mathrm{nm}\left(t_{1}=30 \mathrm{~nm}\right)$ in Fig. 4 (d). In our simulation, the thickness of $\mathrm{SiO}_{2}$ (BOX) below the $\mathrm{Si}$ was assumed to be infinite. We also verified the cases for finite thicknesses (data not shown), and found that the position, height and width of the main absorption peak barely changed for the thicknesses of $100 \sim 400 \mathrm{~nm}$ and infinity, although small satellite peaks appeared. Therefore, it is expected that the general behavior of the proposed SOI photodiode can be accessed by the simple structure with infinite BOX. In Ref. [8], high quantum efficiency of more than $50 \%$, which is a parameter equivalent to the absorption efficiency in this paper, was obtained for 250-nm-thick indium germanium arsenide (InGaAs) MSM photodiode with Au SP antenna of slit array. Moreover, by utilizing the Au SP antenna and an indium phosphide / indium germanium arsenic phosphide (InP/InGaAsP) distributed Bragg reflector (DBR), the quantum efficiency was surprisingly increased to about $95 \%$ at the wavelength of $1550 \mathrm{~nm}$ due to the enhanced

confinement effect of the light power into the InGaAs absorption layer. In contrast to the compound semiconductor devices, it is rather difficult to implement DBR in Si-based devices due to the limited choice of lattice-matched epitaxial materials. However, we made the best use of SOI material by optimizing the Au SP antenna structure to attain the high absorption efficiency. Note that our antenna is not completely slitted after optimization, and therefore the SP modes are different from those in Ref. [8].

\section{B. Optimized Si Thickness for Single-Peaked Absorption}

Figure 5 shows the binary distributions of the absorption efficiency in the wavelength and the Si thickness $t_{\mathrm{SOI}}$ for the cases with and without the $\mathrm{Au} \mathrm{L} / \mathrm{S}$ grating. White area corresponds to the absorption efficiency exceeding $20 \%$. Overall appearance of Fig. 5 does not change for threshold value from 10 to $25 \%$, but the loci in the long wavelength region become invisible for large threshold value, and those in the short wavelength and thick SOI region become whitewashed for small threshold value. Without the grating [Fig. 5(a)], the white area is located at shorter wavelength and thicker Si thickness. Note that the stripes appear due to the light interferences in the Si layer. On the other hand, with the grating [Fig. 5(b)], the white stripes are extended to the longer wavelength and the thinner Si thickness region. The number of absorption peaks along the wavelength increases with the $t_{\mathrm{SOI}}$. For the photodetector with the single-peaked absorption characteristics, the $t_{\mathrm{SOI}}$ should be thinner than $110 \mathrm{~nm}$.

\section{Resonances at absorption peak}

Without Au SP antenna, the SOI-MOS photodiode can obtain only the resonance due to the interferences of the lights reflected from the material interfaces. In the case where the SOI thickness is $100 \mathrm{~nm}$, the peak wavelength of absorption efficiency appears at $450 \mathrm{~nm}$ as shown in Fig. 4. However, the $\mathrm{Au} \mathrm{SP}$ antenna with L/S grating works not only as resonator but also as scatterer to various directions for incident light. By these effects, the horizontal resonant modes can exist in the SOI-MOS photodiode. In order to clarify the resonance inside the SOI at the peak wavelength of the enhanced light absorption in Fig. 4, the spatial distributions of magnetic field and Poynting vector are calculated for the representative structural parameters in Fig. 4 ( $p=200 \mathrm{~nm}, w=140 \mathrm{~nm}, t_{1}=30$ $\mathrm{nm}, t_{2}=80 \mathrm{~nm}$ ). Figure 6 shows the magnetic field $H_{y}$ distribution at the two different wavelengths of absorption peak in the Au SP antenna. The result corresponding to the first peak at the wavelength of $433 \mathrm{~nm}$ is shown in Fig. 6(a). Inside SOI, the numbers of the vertical and the horizontal anti-nodes are two and two, respectively. If there are horizontal anti-nodes in the resonant state, the peak wavelength is directly changed with the change in the grating period $p$ as shown in Fig. 4(a). At the second peak of the wavelength of $550 \mathrm{~nm}$ [Fig. 6(b)], the numbers of the vertical and the horizontal anti-nodes are one and two, respectively. Thus, the vertical resonance is the fundamental mode. As the absorption efficiency becomes maximum in this resonance condition, the flow of 

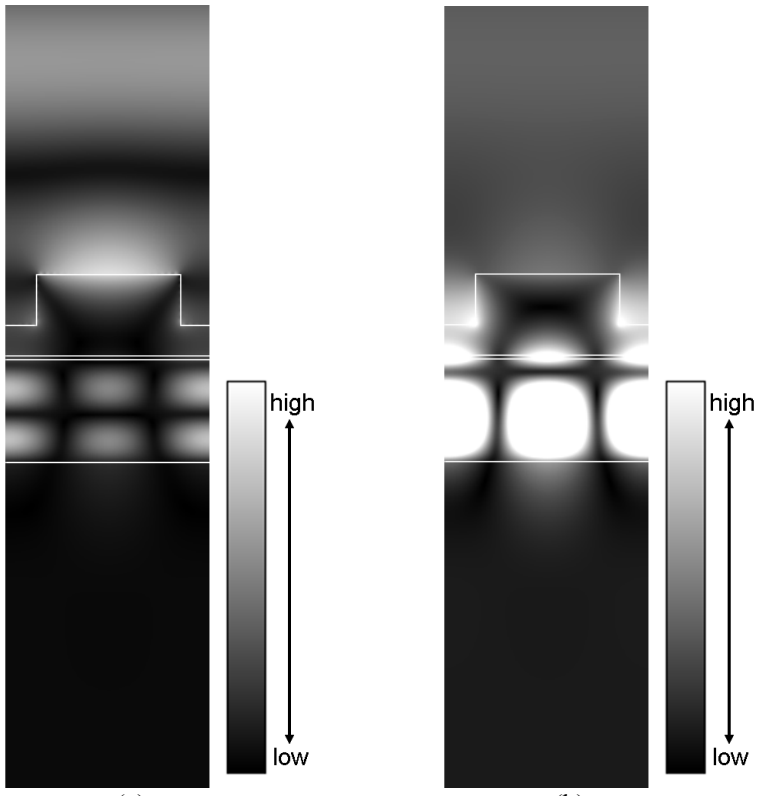

(a)

(b)

Fig. 6. Magnetic field $H_{y}$ distributions at the wavelengths of absorption peak for the Au SP antenna with $p=200 \mathrm{~nm}, w=140 \mathrm{~nm}, t_{1}=30 \mathrm{~nm}$, and $t_{2}=80 \mathrm{~nm}$ on 5-nm-thick $\mathrm{SiO}_{2}, 100$-nm-thick $\mathrm{Si}$, and infinite $\mathrm{SiO}_{2}$ substrate. Incident wavelengths are (a) $433 \mathrm{~nm}$ and (b) $550 \mathrm{~nm}$. Note that the intensity values corresponding to the brightness in (a) and (b) are coincided.

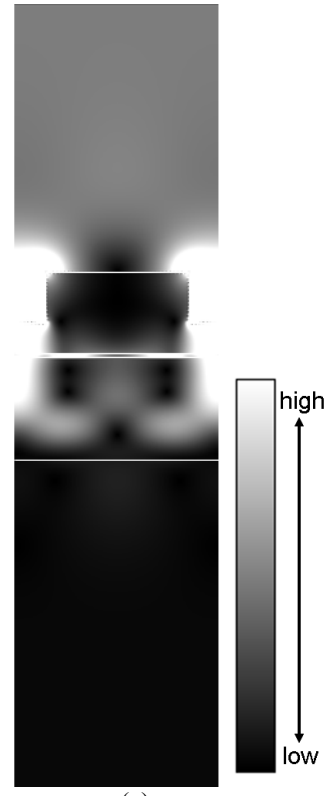

(a)

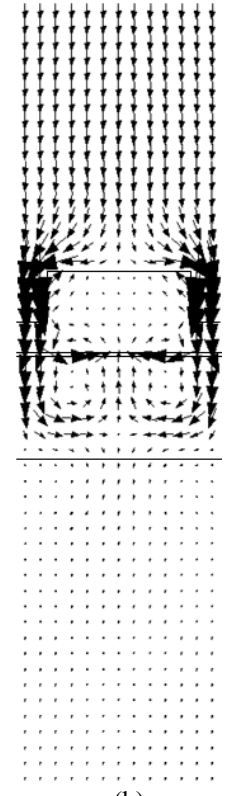

(b)
Fig. 7. Distributions of (a) the magnitude of Poynting vector and (b) Poynting vector at the peak wavelength of the light absorption (i.e. $550 \mathrm{~nm}$ ) for the Au SP antenna with $p=200 \mathrm{~nm}, w=140 \mathrm{~nm}, t_{1}=30 \mathrm{~nm}$ and $t_{2}=80 \mathrm{~nm}$ on 5-nm-thick $\mathrm{SiO}_{2}, 100$-nm-thick $\mathrm{Si}$ and infinite $\mathrm{SiO}_{2}$ substrate.

electromagnetic energy is analyzed using Poynting vector distribution in Fig. 7. Incident light enters the SOI mainly from the thinner Au region. The entering light is mostly terminated at the central bottom of the thicker Au. This result indicates that the absorption efficiency can be enhanced due to the resonance in the SOI assisted by SP excitation around the antenna.

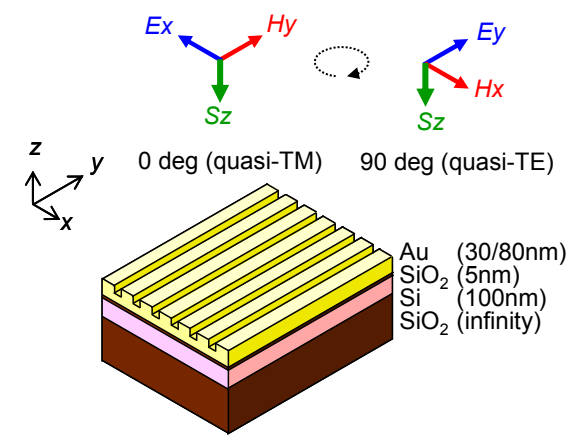

Fig. 8. 3D structure of the SOI-MOS photodetector with Au L/S grating, and the definition of polarization angle.

TABLE I

SIMULATION CONDITIONS FOR POLARIZATION DEPENDENCE.

\begin{tabular}{ll}
\hline \multicolumn{1}{c}{ Parameter } & \multicolumn{1}{c}{ Value } \\
\hline descretized interval & $\Delta d=5 \mathrm{~nm}$ \\
descretized time step & $\Delta t=\Delta d /(2 c)=8.34 \times 10^{-18} \mathrm{~s}$ \\
grating period & $p=200 \mathrm{~nm}$ \\
grating width & $w=140 \mathrm{~nm}$ \\
gold thicknesses & $t_{1}=30 \mathrm{~nm}, t_{2}=80 \mathrm{~nm}$ \\
SOI thickness & $t_{\text {SOI }}=100 \mathrm{~nm}$ \\
incident wavelength & $\lambda=550 \mathrm{~nm}$ \\
incident beam & $600 \times 600 \mathrm{~nm}^{2}$ \\
& $($ uniform intensity) \\
height of incident plane & $2000 \mathrm{~nm}$ \\
$\quad$ from the top of SOI layer & \\
observation plane & $1000 \times 1000 \mathrm{~nm}^{2}$ \\
observation window & $17.55 \sim 24.89 \times 10^{-15} \mathrm{~s}$ \\
\hline \hline
\end{tabular}

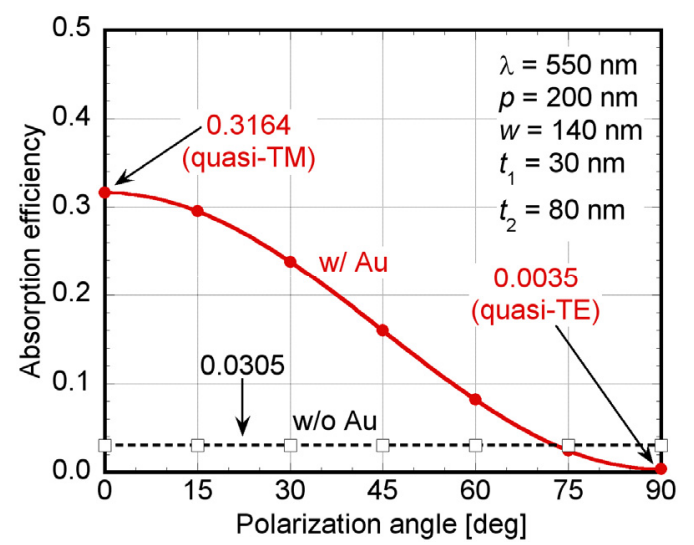

Fig. 9. Dependence of silicon absorption efficiency on polarization angle for the 3D structure in Fig.8. Fitting curve is based on cosine function.

\section{Polarization Response by $3 D$ Simulation}

In order to analyze the dependence of the absorption efficiency on polarization, a 3D structure in Fig. 8 is analyzed. The polarization angle is also defined in Fig. 8. The simulation conditions are summarized in Table 1 . Here, the height of the incident plane of $2000 \mathrm{~nm}$ does not seem sufficiently high, considering the wavelength of $550 \mathrm{~nm}$. However, in order to obtain planar wavefront in the vicinity of the antenna surface, it is preferable to set the height of the light source rather low in this particular configuration. Figure 9 shows the silicon 
absorption efficiency as a function of the polarization angle. The absorption efficiency of $32 \%$ at zero deg, corresponding to the quasi-transverse magnetic (quasi-TM) polarization, is close to the $37 \%$ obtained by $2 \mathrm{D}$ simulation with perfectly planar wave in the section III A, partially supporting the validity of the 3D simulation conditions. The absorption efficiency decreases with increasing polarization angle. The absorption efficiencies at zero polarization angle coincide well with those of 2D simulation [see Fig. 4(a) for $p=200 \mathrm{~nm}$ ], indicating that the effect of finite sizes of the SP antenna and incident beam is not conspicuous under the conditions shown in Table 1. Figure 9 indicates that the photodetector with $\mathrm{Au} \mathrm{L} / \mathrm{S}$ gratings can discriminate the incident lights with different polarizations. As mentioned in the previous section, SP modes can be excited only in transverse magnetic (TM) polarization. However, the absorption efficiency for quasi-transverse electric (quasi-TE) mode is not zero as shown in Fig. 9, because weak optical power of quasi-TE component can be introduced in the SOI layer thorough the thinner part of the Au grating structure even without the generation of SP modes. Note that such leakage of the optical power is inevitable, because the thinner part is essential to provide coupling between SP modes on the front and back surfaces of the Au grating. In order to evaluate the performance as a polarization detector, the rejection ratio [= $\gamma_{\mathrm{TM}} / \gamma_{\mathrm{TE}}$, where $\gamma_{\mathrm{TM}}$ and $\gamma_{\mathrm{TE}}$ are the absorption efficiencies for quasi-TM component and quasi-TE component, respectively] is introduced. In the present condition, the rejection ratio can be as high as 90. Compared with the case without $\mathrm{Au} \mathrm{L/S} \mathrm{grating,}$ the absorption efficiency can be enhanced for the polarization angle less than $72 \mathrm{deg}$.

\section{CONCLUSION}

We demonstrated the enhanced light absorption of SOI-MOS photodetector with SP antenna of Au L/S grating. It was shown the grating structure could be optimized especially for high absorption efficiency and single-peaked spectroscopic characteristics. The grating did not only enhance the light absorption up to $37 \%$ for 100 -nm-thick Si at the wavelength of $550 \mathrm{~nm}$, but also worked as a filter whose peak wavelength could be tailored from 480 to $620 \mathrm{~nm}$ by changing its period. The latter result suggested that photodetectors with different absorption wavelengths could be integrated in a chip just by preparing Au L/S gratings with different periods. Furthermore, it was shown that the Si layer thinner than $110 \mathrm{~nm}$ was needed for single-peaked absorption characteristics. In addition, we clarified the resonant state at the wavelengths of the peak absorption efficiency by utilizing the magnetic field distribution and the flows of the Poynting vector. We also extended the analysis to $3 \mathrm{D}$ structures to investigate the polarized light response of the SOI-MOS photodetector with SP antenna. It was shown that polarization of the incident light could be discriminated by altering the grating direction with rejection ratio as high as 90 . These results open up the possibility of SOI-MOS photodetectors featuring high-speed operation, high voltage gain, and unique functionalities such as wavelength and polarization filtering.

\section{REFERENCES}

[1] T.W. Ebbesen, H.J. Lezec, H.F. Ghaemi, T. Thio and P.A. Wolf, "Extraordinary optical transmission through sub-wavelength hole arrays," Nature, 391, pp.667-669, Feb. 1998.

[2] H. F. Ghaemi, Tineke Thio, D. E. Grupp, T. W. Ebbesen, and H. J. Lezec, "Surface plasmons enhance optical transmission through subwavelength holes," Phys. Rev. B, vol.58, no.11, pp.6779-6782, Sep. 1998.

[3] L. Martín-Moreno, F. J. García-Vidal, H. J. Lezec, K. M. Pellerin, T. Thio, J. B. Pendry, and T.W. Ebbesen, "Theory of extraordinary optical transmission through subwavelength hole arrays," Phys. Rev. Lett., vol.86, no.6, pp.1114-1117, Feb. 2001

[4] D.M. Schaadt, B. Feng, and E.T. Yub "Enhanced semiconductor optical absorption via surface plasmon excitation in metal nanoparticles," Appl. Phys. Lett., vol.86, 063106, 2005.

[5] C.L. Tan, V.V. Lysak, K. Alameh, Y.T. Lee "Absorption enhancement of $980 \mathrm{~nm}$ MSM photodetector with a plasmonic grating structure," Opt. Comm., vol.283, no.9, pp.1763-1767, 2010.

[6] D. Crouse, "Numerical modeling and electromagnetic resonant modes in complex grating structure and optoelectronic device applications," IEEE Trans. Electron Devices, vol.52, no.44, pp.2365-2373, Nov. 2005.

[7] T. Ishi, J. Fujikata, K. Makita, T. Baba and K. Ohashi, "Si nano-photodiode with a surface plasmon antenna," Jpn. J. Appl. Phys., vol.44, no.12 pp.L364-L366, Mar. 2005.

[8] D. Okamoto, J. Fujikata, K. Nishi, and K. Ohashi, "Numerical study of near-infrared photodetectors with surface-plasmon antenna for optical communication,” Jpn. J. Appl. Phys., vol. 47, no.4, pp.2921-2923, April 2008.

[9] J. Hetterich, G. Bastian, N. A. Gippius, S. G. Tikhodeev, G. von Plessen, and U. Lemmer, "Optimized Design of Plasmonic MSM Photodetector," IEEE J. Quant. Electron., vol.43, no.10, pp.855-859, Oct. 2007.

[10] A. Taflove and S.C. Hagness, Computational Electrodynamics -The Finite Difference-Time Domain Method-, 3rd ed., Artech House, MA, 2005.

[11] http://www.sopra-sa.com/index.php

[12] H. Satoh, Y. Matsuo, H. Inokawa, and A. Ono, "Evaluation of Adhesion Materials for Gold Line-and-Space Surface Plasmon Antenna on SOI-MOS Photodiode," Proc. 2010 Silicon Nanoelectronics Workshop (SNW2010), p.131, 2010.

Hiroaki Satoh (S'01-M'04) received the B.E. degree from Muroran Institute of Technology, Hokkaido, Japan, in 1999, and the M.E. and the Ph.D degrees from Hokkaido University, Hokkaido, Japan, in 2001 and 2004, respectively. He was a Research Associate of the University of Tokushima, Tokushima, Japan, from 2004 to 2007. He became an Assistant Professor of the Research Institute of Electronics, Shizuoka University, Hamamatsu, Japan, in 2007. He has been engaged in research on the computational electromagnetics, and its application to silicon nanodevices for advanced photonics.

Dr. Satoh is a member of the Japan Society of Applied Physics, the Institute of Electronics, Information and Communication Engineers of Japan, and the Institute of Electrical Engineers of Japan.

Hiroshi Inokawa (M'90) received the Ph.D. degree in electrical engineering from Kyoto University, Kyoto, Japan, in 1985. In the same year, he joined the Atsugi Electrical Communications Laboratories, Nippon Telegraph and Telephone Corporation (NTT), Kanagawa, Japan. Since then, he has been engaged in research and development of scaled-down CMOS devices and silicon single-electron devices. In 2006, he became a professor of the Research Institute of Electronics, Shizuoka University, Hamamatsu, Japan, where he has been studying nanodevices for advanced circuits and systems.

Prof. Inokawa is a member of the Institute of Electrical and Electronics Engineers, the Japan Society of Applied Physics, the Institute of Electronics, Information and Communication Engineers of Japan, and the Institute of Electrical Engineers of Japan. 